\title{
PRKACA mutations in cortisol-producing adenomas and adrenal hyperplasia: a single-center study of $\mathbf{6 0}$ cases
}

\author{
Anne Thiel ${ }^{1}$, Anna-Carinna Reis ${ }^{2,3}$, Matthias Haase ${ }^{4}$, Gerald Goh ${ }^{5,6}$, \\ Matthias Schott ${ }^{4}$, Holger S Willenberg ${ }^{4,7}$ and Ute I Scholl ${ }^{1}$
}

Departments of ${ }^{1}$ Nephrology and ${ }^{2}$ Pathology, School of Medicine, Heinrich Heine University Düsseldorf, Moorenstraße 5, 40225 Düsseldorf, Germany, ${ }^{3}$ Department of Pathology, University Hospital Essen, 45147 Essen, Germany, ${ }^{4}$ Division of Specific Endocrinology, School of Medicine, Heinrich Heine University Düsseldorf, Moorenstraße 5, 40225 Düsseldorf, Germany, ${ }^{5}$ Department of Genetics, Yale University School of Medicine, New Haven, Connecticut 06520, USA, ${ }^{6}$ University College London Cancer Institute, London WC1E 6BT, UK and ${ }^{7}$ Division of Endocrinology and Metabolic Disease, University Medical Center, 18057 Rostock, Germany

\author{
Correspondence \\ should be addressed \\ to U I Scholl \\ Email \\ ute.scholl@med. \\ uni-duesseldorf.de
}

\section{Abstract}

Objective: Cortisol excess due to adrenal adenomas or hyperplasia causes Cushing's syndrome. Recent genetic studies have identified a somatic PRKACA ${ }^{L 206 R}$ mutation as a cause of cortisol-producing adenomas. We aimed to compare the clinical features of PRKACA-mutant lesions with those of CTNNB1 mutations, and to search for similar mutations in unilateral hyperplasia or tumors co-secreting aldosterone.

Design, patients, and methods: In this study, 60 patients with cortisol excess who had adrenalectomies at our institution between 1992 and 2013 were assessed, and somatic mutations were determined by Sanger sequencing. A total of 36 patients had overt Cushing's syndrome, the remainder were subclinical: 59 cases were adenomas (three bilateral) and one was classified as hyperplasia. Four tumors had proven co-secretion of aldosterone.

Results: Among cortisol-secreting unilateral lesions without evidence of co-secretion $(n=52)$, we identified somatic mutations in PRKACA (L206R) in 23.1\%, CTNNB1 (S45P, S45F) in 23.1\%, GNAS (R201C) in 5.8\%, and CTNNB1+GNAS (S45P, $\mathrm{R} 201 \mathrm{H})$ in $1.9 \%$. PRKACA and GNAS mutations were mutually exclusive. Of the co-secreting tumors, two (50\%) had mutations in KCNJ5 (G151R and L168R). The hyperplastic gland showed a PRKACA ${ }^{\text {L206R }}$ mutation, while patients with bilateral adenomas did not have known somatic mutations. PRKACA-mutant lesions were associated with younger age, overt Cushing's syndrome, and higher cortisol levels vs non-PRKACA-mutant or CTNNB1-mutant lesions. CTNNB1 mutations were more significantly associated with right than left lesions.

Conclusions: PRKACA ${ }^{L 206 R}$ is present not only in adenomas, but also in unilateral hyperplasia and is associated with more severe autonomous cortisol secretion. Bilateral adenomas may be caused by yet-unknown germline mutations.

\section{Introduction}

The steroid hormone cortisol is produced by the adrenal zona fasciculata and acts to increase gluconeogenesis and regulate inflammatory activity. Physiologically, the release of hypothalamic corticotropin-releasing hormone $(\mathrm{CRH})$ induces secretion of corticotropin (ACTH) from the pituitary gland. The binding of ACTH to its G-protein-coupled melanocortin 2 receptor (c) 2015 European Society of Endocrinology Printed in Great Britain activates adenylyl cyclase, leading to cAMP production. cAMP binds to the regulatory subunit of protein kinase $\mathrm{A}$ (PKA), causing the release of the catalytic subunit, which phosphorylates downstream targets that act to increase cortisol production and cell proliferation (1). A regulatory feedback mechanism prevents excess production of cortisol. 
Autonomous production of CRH, ACTH, or cortisol causes endogenous Cushing's syndrome (2). Recently, recurrent somatic mutations in cortisol-producing adenomas have been identified by next-generation sequencing and confirmed in follow-up studies $(3,4,5,6,7,8)$. The most frequently mutated gene $(35.4 \%$, range $14.3-65.5 \%$ across published cohorts, Supplementary Table 1, see section on supplementary data given at the end of this article) is PRKACA, and almost all mutations are accounted for by a single p.Leu206Arg (L206R) substitution in the encoded catalytic subunit of PKA. The mutation abolishes binding to the regulatory subunit and causes constitutive PKA activity, excess cortisol biosynthesis, and cell proliferation. Somatic hotspot mutations in the CTNNB1 gene, encoding $\beta$-catenin, were found in $16.4 \%$ of tumors in one study (6), while the remaining authors did not systematically screen for such mutations (Supplementary Table 1). Activating CTNNB1 mutations occur not only in adrenal adenomas, including hormonally inactive tumors, but also in malignancies of the adrenal gland and other organs. These activate Wnt signaling by preventing $\beta$-catenin degradation, thereby causing proliferation, but any role in cortisol production remains to be determined $(9,10)$. In mice, constitutive activation of $\beta$-catenin causes adrenal hyperplasia, the development of malignant characteristics, and, interestingly, primary aldosteronism in the absence of hypercortisolism (11). Mutations in GNAS, the stimulatory G-protein $\alpha$-subunit associated with the melanocortin receptor 2 , were initially discovered to be the cause of macronodular adrenal hyperplasias (12) and are less frequent causes of cortisol-producing adenomas (6). Mutations in GNAS and PRKACA are mutually exclusive and appear to be associated with small tumors, young age at presentation, and overt Cushing's syndrome (6).

The prevalence of hypertension in patients with Cushing's syndrome is $\sim 80 \%$. The factors that are thought to contribute to the hypertensive effects of cortisol excess include the mineralocorticoid activity of cortisol, activation of the renin-angiotensin system, enhanced vasoconstriction and blunted vasodilatation $(13,14)$. In rare cases, secretion of aldosterone is the main cause of hypertension and optional hypokalemia, and co-secretion of cortisol may play a minor role. Some of the corresponding tumors have been shown to carry activating mutations in the KCNJ5 potassium channel gene that are specific to aldosterone-producing adenomas $(15,16,17)$. Only one study screened for PRKACA mutations in these tumors, but did not report any (7). Similarly, no PRKACA mutations were found in 20 'pure' aldosterone-secreting adenomas (3).
To compare the features of subjects with PRKACA mutations vs CTNNB1 mutations, in this study, we report the clinical and genetic characteristics of 60 patients with cortisol excess, who underwent adrenalectomy at our institution. Using Sanger sequencing, we confirm a high frequency of PRKACA mutations in cortisol-producing adenomas, but not in those co-secreting aldosterone. We show that PRKACA mutations are associated with a more severe clinical phenotype. Unexpectedly, we demonstrate the presence of a PRKACA mutation in unilateral adrenal hyperplasia.

\section{Subjects and methods}

\section{Subjects and clinical data}

Formalin-fixed paraffin-embedded (FFPE) tissue samples of 60 patients who underwent unilateral or bilateral adrenalectomy for Cushing's syndrome (11 with previously published genetic data (6) and two previously published as case reports (15)) at University Hospital Düsseldorf between 1992 and 2013 were analyzed. Matched normal tissue samples were available in 53 cases.

Hypercortisolism was diagnosed based on a combination of biochemical test results (elevated urinary free cortisol, increased late-night salivary or serum cortisol, suppressed plasma ACTH levels, nonsuppressible serum cortisol levels) and typical signs of hypercortisolism (e.g. muscle weakness, skin fragility and osteoporosis). Overt Cushing's syndrome was defined as either the presence of at least three abnormal biochemical test results or as typical signs plus at least two abnormal biochemical test results. Subclinical Cushing's syndrome was defined as at least one abnormal biochemical test result in the absence of clinical signs $(3,8)$.

Dexamethasone suppression tests typically consisted of a 1-2 mg overnight dose, with cortisol measured the following morning, and were used as a screening tool for nonsuppressible Cushing's syndrome (overt or subclinical). In rare cases, $8 \mathrm{mg}$ dexamethasone tests were performed, which were considered indicative of adrenal Cushing's syndrome in case of nonsuppression of cortisol in the presence of suppressed ACTH.

Co-secretion of aldosterone was assumed based on elevated aldosterone levels in the presence of suppressed renin and confirmed by salt infusion testing, fludrocortisone suppression testing, and/or normalization of values after surgery. The patient characteristics are summarized in Supplementary Table 2, see section on supplementary data given at the end of this article. 
All specimens were independently reviewed by two pathologists and categorized by size and histology (absence of signs of malignancy, adenoma vs hyperplasia). Written informed consent was obtained before adrenalectomy, and the study was approved by the Institutional Review Board at the School of Medicine, Heinrich Heine University Düsseldorf.

\section{DNA extraction, PCR, and sequencing}

H\&E staining was performed according to standard procedures in a clinical pathology laboratory. Areas of tumor and normal tissue were marked by a pathologist, and two $2 \mathrm{~mm}$ cores were obtained from each area. DNA was isolated using the BiOstic FFPE Tissue DNA Isolation Kit (MO BIO Laboratories, Carlsbad, CA, USA), repaired with the PreCR Repair Mix (New England Biolabs, Beverly, MA, USA), and purified with the Agencourt Ampure XP PCR Purification System (Agencourt Bioscience, Beverly, MA, USA) according to the manufacturers' instructions.

Routine PCR amplification of known target genes from matched tumor and normal samples was performed using specific primers. As larger template regions failed to amplify, shorter template regions were amplified. The primer sequences are listed in Supplementary Table 3, see section on supplementary data given at the end of this article. PCR purification and direct bidirectional Sanger sequencing were performed (Beckman Coulter Genomics, Takely, Essex, UK). All identified mutations were confirmed in independent PCRs.

\section{Targeted capture and whole-exome sequencing}

Targeted capture was performed at the Yale Center for Genome Analysis using the NimbleGen 2.1 Exome reagent, followed by sequencing on the Illumina HiSeq platform as previously described (6), and sequences were aligned to the human genome (hg19).

\section{Statistical analysis}

Data are shown as mean \pm s.D., unless otherwise indicated. Comparisons between two groups were performed using two-tailed Fisher's exact test or Mann-Whitney $U$ tests, and $P$ values $<0.05$ were considered to be significant.

\section{Results}

\section{Characterization of the cohort}

Among the 60 adrenalectomies for adrenal Cushing's syndrome (Fig. 1 and Supplementary Table 2), 57 were

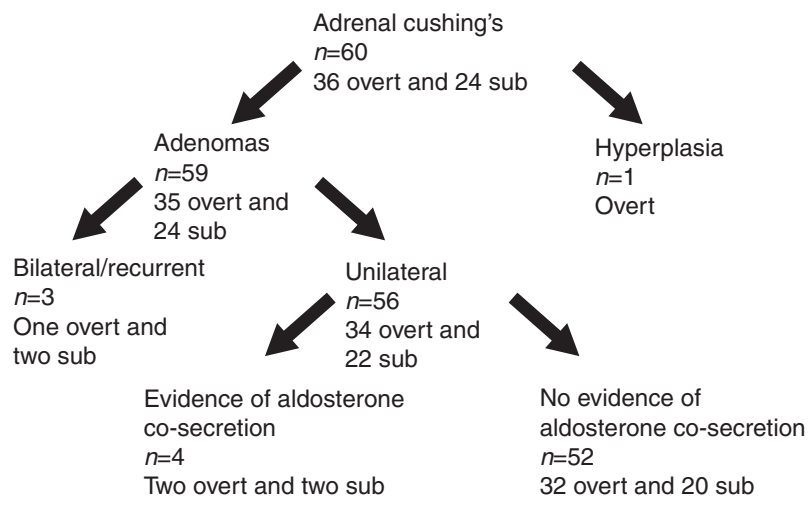

Figure 1

Overview of the cohort. Overt, overt Cushing's syndrome; sub, subclinical Cushing's syndrome.

unilateral (40 left and 17 right) and three were bilateral (one case with simultaneous bilateral adrenalectomy due to bilateral adenomas, two subsequent right and left adrenalectomies due to recurrence on the contralateral side). Between 1992 and 1997, typically, open adrenalectomies were performed, with a switch to laparoscopic surgery in 1997 and retroperitoneoscopic surgery in 2007. Pathology revealed single or multiple adrenal adenomas in 59 cases (two with associated nodular hyperplasia) and a single case of unilateral diffuse adrenal hyperplasia (Fig. 2 and Supplementary Table 2).

Among the 60 subjects, 36 presented with overt Cushing's syndrome, while 24 individuals were subclinical. In four cases (CS057-CS060), co-secretion of aldosterone was noted. Previous studies suggest a distinct, aldosterone-producing adenoma-like biology of tumors co-secreting cortisol and aldosterone (16). Similarly, we reasoned that bilateral or recurrent adenomas may be due to yet unknown germline mutations. Thus, these two groups were analyzed separately.

\section{PRKACA and CTNNB1 mutations are frequent in cortisol-secreting tumors; PRKACA mutations are associated with a more severe phenotype}

To determine the frequencies of known somatic mutations in patients with unilateral cortisol-secreting adenomas without evidence of aldosterone co-secretion $(n=52)$, we performed PCR and direct Sanger sequencing of PRKACA (exon 7, encoding the L206R mutation $(3,4,5,6)$ ), CTNNB1 (exon 3 with hotspot mutations $(6,9)$ ), and GNAS (exons 8 and 9 with known disease-causing mutations (6)) (Supplementary Table 2 and Table 1). As available 
CS041 - hyperplasia, PRKACA $A^{\text {L206R }}$
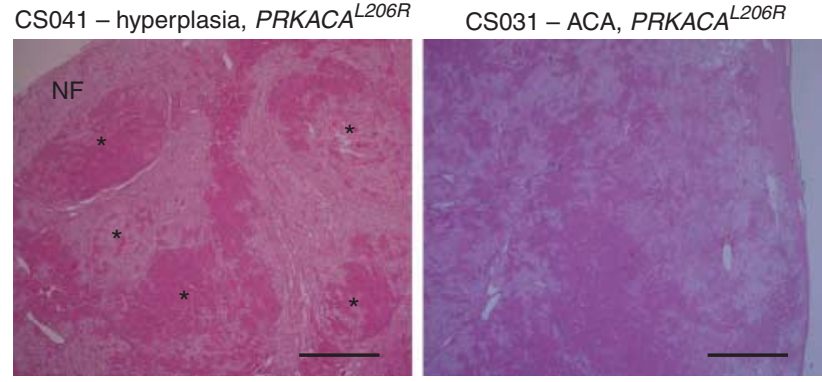

\section{Figure 2}

Histology of samples with PRKACA ${ }^{L 206 R}$ mutation (H\&E). Left, sample CS041 with elements of normal fasciculata (NF), in addition rearrangement of zona fasciculata and reticularis and several hyperplastic micronodules (*). Right, sample CS031 with adrenocortical adenoma (ACA) shows typical areas of lipid-rich clear cells and oncocytic cells (eosinophilic). Scale bars, $1000 \mu \mathrm{m}$.

(46 samples), normal tissue samples were screened for the same mutations. With the exception of an extremely low peak in sample CSO48 that was assumed to be tumor contamination, all mutations were confirmed to be somatic, i.e. absent from normal tissue. The age of patients at time of surgery was $49.8 \pm 13.4$ (s.D., range of 28-74) years. The mean tumor size was $3.6 \pm 1.1 \mathrm{~cm}$ (range of 1.4-6), and the male:female ratio was 3:49. Thirty-two patients (61.5\%) presented with overt Cushing's syndrome. Serum cortisol was $20.0 \pm 8.0 \mu \mathrm{g} / \mathrm{dl}$, urinary cortisol was $315.2 \pm 318.5 \mu \mathrm{g} / 24-\mathrm{h}$, and ACTH was $4.2 \pm 4.2 \mathrm{pg} / \mathrm{ml}$. After dexamethasone suppression, serum cortisol was $12.5 \pm 10.4 \mu \mathrm{g} / \mathrm{dl}$.

Twelve patients $(23.1 \%)$ carried the previously described c.617T $>\mathrm{G}$ (p.Leu206Arg) missense mutation in PRKACA. Another 12 subjects (23.1\%) had a CTNNB1 mutation of codon 45 (eight cases with c.133T $>\mathrm{C}$ (p.Ser45Pro); four instances of c.134C $>\mathrm{T}$ (p.Ser45Phe)). In three patients (5.8\%), a c.601C $>\mathrm{T}$ (p.Arg201Cys) mutation in GNAS was found, and one patient (1.9\%) had concomitant CTNNB1 and GNAS mutations (c.133T > C (p.Ser45Pro) and c.602G >A (p.Arg201His) respectively) (Fig. 3 and Table 1).

Within this group, PRKACA mutations were associated with a significantly more severe phenotype (Table 1 and Fig. 4A, B and C). Patients presented at a significantly younger age than those without PRKACA mutations $(37.7 \pm 7.7$ years vs $53.5 \pm 12.6$ years $(P<0.0001))$ or with CTNNB1 mutations $(59.6 \pm 10.9$ years, $P<0.0001$ vs $P R K A C A)$. Serum cortisol levels after dexamethasone suppression in those with PRKACA mutations were significantly higher $(24.6 \pm 3.8 \mu \mathrm{g} / \mathrm{dl}$ vs $9.0 \pm 8.9 \mu \mathrm{g} / \mathrm{dl}$ in those without PRKACA mutations $(P<0.0001)$ and $9.6 \pm 11.8 \mu \mathrm{g} / \mathrm{dl}$ in those with CTNNB1 mutations $(P=0.0119$ vs $P R K A C A))$.

All three male subjects in the cohort had CTNNB1 mutations (one combined with GNAS mutation).

Subjects with PRKACA mutation had overt Cushing's syndrome in $11 / 12$ cases, vs $21 / 40$ of the remainder $(P=0.0181)$, and $5 / 12$ with CTNNB1 mutation only $(P=0.0272$ vs $P R K A C A)$. Whereas PRKACA mutations were enriched in the subcohort with overt Cushing's syndrome ( $34.4 \%$ vs $23.1 \%$ overall), the opposite was the case for CTNNB1 mutations ( $15.6 \%$ vs $25.0 \%$ overall).

\section{PRKACA is mutated in a sample with diffuse hyperplasia}

Remarkably, one of the somatic PRKACA mutations occurred in the single sample with apparently unilateral adrenal hyperplasia (CS041), a rare subgroup of adrenal Cushing's syndrome that has not been previously described to carry such mutations (Fig. 2 and Supplementary Table 2). Upon independent reassessment of several areas of the affected glands, this mutation was confirmed to be present in four hyperplastic areas, but absent from non-hyperplastic tissue (Supplementary Figure 1, see section on supplementary data given at the end of this article). No germline mutations in genes previously described to cause macronodular adrenal hyperplasia, and specifically, no ARMC5 or PRKAR1A mutations were identified by targeted capture and sequencing.

The subject with unilateral adrenal hyperplasia was evaluated for secondary hypertension at age 49 years. Markedly increased urinary cortisol $(626 \mu \mathrm{g} / 24 \mathrm{~h})$ was noted, ACTH was suppressed $(<1.0 \mathrm{pg} / \mathrm{ml})$, and urinary tetrahydroaldosterone $(<10 \mu \mathrm{g} / 24 \mathrm{~h})$, serum aldosterone $(25 \mathrm{ng} / \mathrm{l})$, metanephrin, and normetanephrin were normal. Plasma renin activity was not suppressed $(5.47 \mathrm{ng} / \mathrm{ml}$ per $\mathrm{h})$. After $2 \mathrm{mg}$ dexamethasone, serum cortisol remained elevated $(27.5 \mu \mathrm{g} / \mathrm{dl})$. High-dose dexamethasone $(8 \mathrm{mg})$ also failed to suppress cortisol $(26.0 \mu \mathrm{g} / \mathrm{dl})$. Imaging demonstrated a $26 \mathrm{~mm}$ right adrenal lesion, and retroperitoneoscopic right adrenalectomy was performed. No adenoma was identified macroscopically or microscopically. Pathology demonstrated regular differentiation into cortex and medulla. There was focal rearrangement of the fascicular and reticular zones with hyperplasia of the zona reticularis and scanty lymphocytic infiltration (Fig. 2). Baseline serum cortisol $(13.9 \mu \mathrm{g} / \mathrm{dl})$ 
Table 1 Summary of patients with unilateral pure cortisol-producing lesions.

\begin{tabular}{|c|c|c|c|c|c|c|c|}
\hline & $\begin{array}{c}\text { All } \\
(n=52)\end{array}$ & $\begin{array}{c}\text { Subclinical } \\
(n=20)\end{array}$ & $\begin{array}{l}\text { Overt } \\
(n=32)\end{array}$ & $\begin{array}{c}\text { PRKACA } \\
(n=12)\end{array}$ & $\begin{array}{c}\text { Non-PRKACA } \\
(n=40)\end{array}$ & $\begin{array}{l}\text { CTNNB1 } \\
(n=13)^{a}\end{array}$ & $\begin{array}{c}\text { WT } \\
(n=24)\end{array}$ \\
\hline Age at surgery (years) & $49.8 \pm 13.4$ & $56.6 \pm 11.7$ & $45.6 \pm 12.8$ & $37.7 \pm 7.7$ & $53.5 \pm 12.6$ & $59.6 \pm 10.9$ & $50.2 \pm 13.2$ \\
\hline Female $(\%)$ & $49 / 52(94.2)$ & $17 / 20(85.0)$ & $32 / 32(100.0)$ & $12 / 12(100.0)$ & $37 / 40(92.5)$ & $10 / 13(76.9)$ & $24 / 24(100.0)$ \\
\hline Adenoma size $(\mathrm{cm})$ & $\begin{array}{c}3.6 \pm 1.1 \\
(n=49)\end{array}$ & $\begin{array}{c}3.8 \pm 1.1 \\
(n=18)\end{array}$ & $\begin{array}{c}3.5 \pm 1.1 \\
(n=31)\end{array}$ & $\begin{array}{c}3.5 \pm 0.8 \\
(n=12)\end{array}$ & $\begin{array}{c}3.6 \pm 1.2 \\
(n=37)\end{array}$ & $\begin{array}{c}3.9 \pm 0.7 \\
(n=12)\end{array}$ & $\begin{array}{c}3.6 \pm 1.3 \\
(n=23)\end{array}$ \\
\hline Left $(\%)$ & $37 / 52(71.2)$ & $17 / 20(85.0)$ & $24 / 32(75.0)$ & $9 / 12(75.0)$ & $28 / 40(70.0)$ & $4 / 13(30.8)$ & $21 / 24(87.5)$ \\
\hline sCortisol ( $\mu \mathrm{g} / \mathrm{dl})$ & $\begin{array}{l}20 \pm 8.0 \\
(n=45)\end{array}$ & $\begin{array}{c}19.3 \pm 7.2 \\
(n=17)\end{array}$ & $\begin{array}{c}20.4 \pm 8.5 \\
(n=28)\end{array}$ & $\begin{array}{c}24.7 \pm 4.0 \\
(n=11)\end{array}$ & $\begin{array}{c}18.5 \pm 8.4 \\
(n=34)\end{array}$ & $\begin{array}{c}20.3 \pm 5.8 \\
(n=12)\end{array}$ & $\begin{array}{c}17.4 \pm 8.4 \\
(n=20)\end{array}$ \\
\hline uCortisol $(\mu \mathrm{g} / 24 \mathrm{~h})$ & $\begin{array}{c}315.2 \pm 318.5 \\
(n=33)\end{array}$ & $\begin{array}{c}134.0 \pm 147.1 \\
(n=6)\end{array}$ & $\begin{array}{c}355.5 \pm 333.8 \\
(n=27)\end{array}$ & $\begin{array}{c}372.8 \pm 151.4 \\
(n=10)\end{array}$ & $\begin{array}{c}290.2 \pm 368.8 \\
(n=23)\end{array}$ & $\begin{array}{c}438.4 \pm 339.1 \\
(n=5)\end{array}$ & $\begin{array}{c}197.8 \pm 257.4 \\
(n=15)\end{array}$ \\
\hline $\begin{array}{l}\text { sCortisol after DST } \\
(\mu \mathrm{g} / \mathrm{dl})\end{array}$ & $\begin{array}{c}12.5 \pm 10.4 \\
(n=41)\end{array}$ & $\begin{array}{c}7.0 \pm 4.6 \\
(n=15)\end{array}$ & $\begin{array}{c}15.6 \pm 11.5 \\
(n=26)\end{array}$ & $\begin{array}{c}24.6 \pm 3.8 \\
(n=9)\end{array}$ & $\begin{array}{c}9.0 \pm 8.9 \\
(n=29)\end{array}$ & $\begin{array}{c}9.6 \pm 11.8 \\
(n=11)\end{array}$ & $\begin{array}{c}8.5 \pm 7.2 \\
(n=18)\end{array}$ \\
\hline ACTH $(\mathrm{pg} / \mathrm{ml})$ & $\begin{array}{c}4.2 \pm 4.2 \\
(n=46)\end{array}$ & $\begin{array}{l}5.1 \pm 3.5 \\
(n=17)\end{array}$ & $\begin{array}{c}3.6 \pm 4.5 \\
(n=29)\end{array}$ & $\begin{array}{l}1.6 \pm 1.4 \\
(n=12)\end{array}$ & $\begin{array}{l}5.1 \pm 4.4 \\
(n=32)\end{array}$ & $\begin{array}{c}4.8 \pm 4.3 \\
(n=12)\end{array}$ & $\begin{array}{l}5.6 \pm 4.8 \\
(n=19)\end{array}$ \\
\hline PRAKACA (\%) & $\begin{array}{c}23.1 \\
(n=12)\end{array}$ & $\begin{array}{c}5.0 \\
(n=1)\end{array}$ & $\begin{array}{c}34.4 \\
(n=11)\end{array}$ & & & & \\
\hline CTNNB1, all ${ }^{\mathrm{a}}(\%)$ & $\begin{array}{c}25.0 \\
(n=13)\end{array}$ & $\begin{array}{c}40.0 \\
(n=8)\end{array}$ & $\begin{array}{l}15.6 \\
(n=5)\end{array}$ & & & & \\
\hline GNAS, all ${ }^{\mathrm{a}}(\%)$ & $\begin{array}{c}7.7 \\
(n=4)\end{array}$ & $\begin{array}{c}5.0 \\
(n=1)\end{array}$ & $\begin{array}{c}9.4 \\
(n=3)\end{array}$ & & & & \\
\hline CTNNB1 + GNAS (\%) & $\begin{array}{c}1.9 \\
(n=1)\end{array}$ & $\begin{array}{c}5.0 \\
(n=1)\end{array}$ & $\begin{array}{c}0.0 \\
(n=0)\end{array}$ & & & & \\
\hline WT (\%) & $\begin{array}{c}46.2 \\
(n=24)\end{array}$ & $\begin{array}{c}55.5 \\
(n=11)\end{array}$ & $\begin{array}{c}40.6 \\
(n=13)\end{array}$ & & & & \\
\hline
\end{tabular}

Subclinical, subclinical Cushing's syndrome; overt, overt Cushing's syndrome; PRKACA, cases with PRKACA mutation; CTNNB1, cases with CTNNB1 mutation; sCortisol, serum cortisol; uCortisol, 24-h urinary cortisol; DST, dexamethasone suppression test; CTNNB1+GNAS, subject with concurrent CTNNB1 and GNAS mutations; WT, subjects without mutations in genes screened.

${ }^{\text {a }}$ One case with CTNNB1 + GNAS mutation.

and ACTH (44.9 pg/ml) became normal 16 months after surgery, and a rise in ACTH and cortisol was observed after $100 \mu \mathrm{g}$ CRH i.v. (ACTH $122.0 \mathrm{pg} / \mathrm{ml}$ after $30 \mathrm{~min}$, $95.2 \mathrm{pg} / \mathrm{ml}$ after $60 \mathrm{~min}$; cortisol $20.9 \mu \mathrm{g} / \mathrm{dl}$ after $30 \mathrm{~min}$, $21.4 \mu \mathrm{g} / \mathrm{dl}$ after $60 \mathrm{~min})$. Computed tomography 3 years after surgery showed some remaining right adrenal tissue and a normal left adrenal gland. Urinary cortisol $(19.5 \mu \mathrm{g} / 24 \mathrm{~h})$ was normal, supporting unilateral hyperplasia as the cause of prior hypercortisolism and arguing against asynchronous macronodular adrenal hyperplasia as the cause of disease.

\section{CTNNB1 is more frequently mutated in the right adrenal gland}

Both cortisol- and aldosterone-producing adenomas occur more frequently on the left side (18), but the underlying reasons remain to be determined. This effect was also seen in our cohort, with $37 / 52$ (71.2\%) of the pure cortisol secreting unilateral lesions occurring on the left side and 9/12 (75.0\%) in the subset with PRKACA mutations. However, unexpectedly, right adrenal adenomas were overrepresented in our samples with CTNNB1 mutations
(9/13 samples with CTNNB1 mutations vs 6/39 without CTNNB1 mutations, $P=0.0006$, Fig. 4D).

\section{Aldosterone and cortisol co-secreting adenomas show mutations in KCNJ5}

In samples co-secreting aldosterone (Supplementary Figure 2, see section on supplementary data given at the end of this article), in addition to the previously mentioned genes, we also sequenced genes known to carry somatic mutations in aldosterone-producing adenomas: KCNJ5 (exon 2 encoding previously described p.Gly151Arg (G151R) and p.Leu168Arg (L168R) mutations (19)), ATP1A1 (exons 4 and $8(20,21)$ ), ATP2B3 (exon 8 (20)), and CACNA1D (exons 6, 8, 8b, 17 and $34(21,22)$ ).

Tumors co-secreting aldosterone and cortisol carried KCNJ5 mutations (G151R and L168R) in 50\% (2/4) cases (Supplementary Table 2). In contrast, none of these patients showed PRKACA mutations, in line with previously published findings $(7,16)$. Mutations in genes other than $K C N J 5$ are considerably less frequent in aldosterone-producing adenomas. Accordingly, we did not detect mutations in $A T P 1 A 1, A T P 2 B 3$, or $C A C N A 1 D$ in this rather small cohort. 
A PRKACA - sample CS003
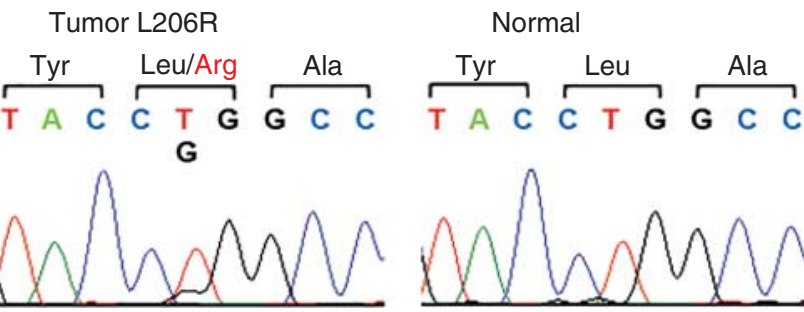

B

CTNNB1 - sample CS002

Tumor S45F Normal
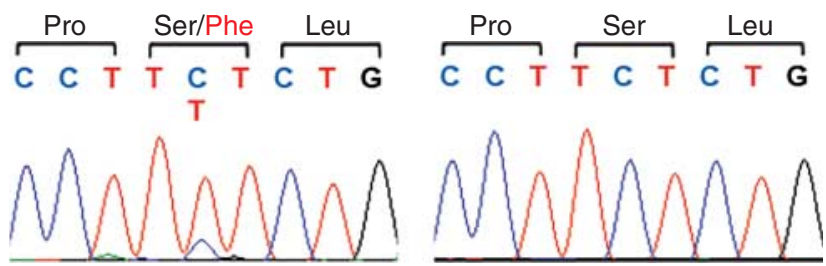

\section{GNAS - sample CS005}

Tumor R201C

Normal
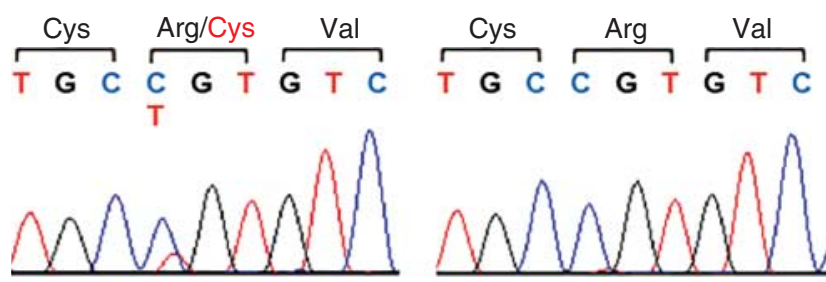

D

KCNJ5 - sample CS059

Tumor L168R

Normal

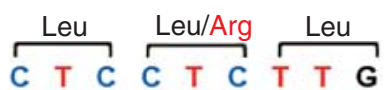

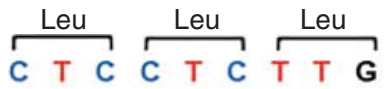
G
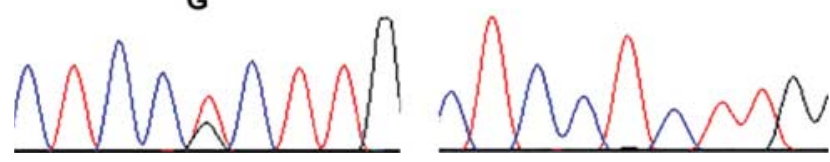

\section{Figure 3}

Somatic mutations in adrenal adenomas associated with Cushing's syndrome. Sanger DNA sequences (representative chromatograms) of tumor and normal DNAs for each gene with mutations in the study cohort. Mutations are not present in normal tissue. (A) PRKACA with heterozygous somatic mutation encoding L206R, (B) CTNNB1 with heterozygous somatic mutations encoding S45F, (C) GNAS with heterozygous somatic mutation encoding R201C, and (D) KCNJ5 with heterozygous somatic mutation encoding L168R. Additional mutations in the same genes and chromosomal positions are listed in Supplementary Table 4.

\section{Bilateral or recurrent adenomas do not carry somatic or germline mutations at typical residues}

None of the bilateral or recurrent adenomas carried mutations in any of the genes examined, pointing to germline mutations as the potential causes of these tumors (Supplementary Table 2). In the case of CSO01 with bilateral adenomas, tumors from both sides were sequenced and tested negative for known mutations. To assess whether bilateral or recurrent adenomas may represent a variant of bilateral macronodular hyperplasia, we performed targeted capture and sequencing of the corresponding germline DNA in all cases. No mutations were detected in genes implicated in adrenal hyperplasia. In particular, all samples tested negative for mutations in ARMC5, PRKAR1A, MEN1, or APC.

\section{Discussion}

In this study, we ascertained an extensive cohort of patients with cortisol-producing adrenal lesions, covering more than 20 years, from a single institution. In this homogenous cohort, we confirmed the high frequency of mutations in PRKACA and CTNNB1 in patients with cortisol-producing adenomas (3, 4, 5, 6). Interestingly, the frequency of PRKACA mutations has shown some variation across different studies. Our finding of PRKACA mutations in $23.1 \%$ of patients with unilateral adenomas producing cortisol only $(34.4 \%$ of patients with overt Cushing's syndrome) is similar to the values reported by Goh et al. (6) (24 and 35\% respectively), but considerably higher frequencies were reported in two Asian studies $(4,5)$ (Supplementary Table 1$)$. In line with previous reports, we show that PRKACA mutations are associated with a more severe phenotype, i.e. higher cortisol levels after dexamethasone suppression as a sign of autonomous cortisol secretion, younger age at presentation, and overt Cushing's syndrome. Only one subject with such mutations was classified as having subclinical Cushing's syndrome. The pathophysiology of the PRKACA $A^{L 206 R}$ mutation, which abolishes binding of the regulatory PKA subunit and provides an autonomous signal for cortisol production, provides a plausible explanation for the severe phenotype observed.

Any role of CTNNB1 mutations in hormone production is less obvious. In our cohort, CTNNB1 mutations are associated with older age at surgery, lower postdexamethasone cortisol levels and lower likelihood of developing overt Cushing's syndrome than PRKACA mutations. Similar or identical mutations have been 
C

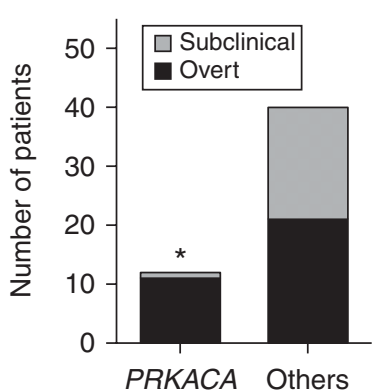

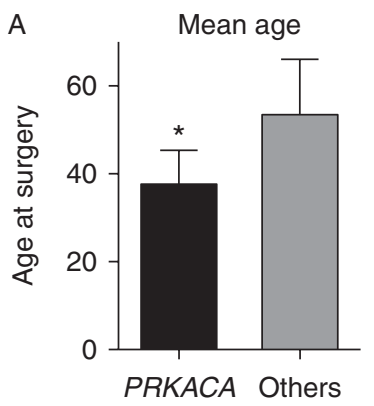

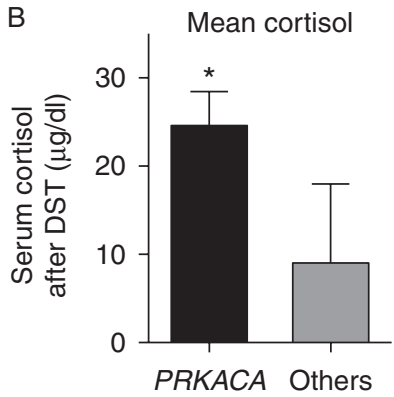

D

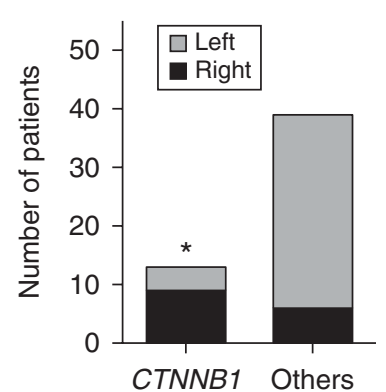

\section{Figure 4}

Clinical correlations of PRKACA and CTNNB1 mutations in unilateral adenomas with cortisol production only. Age at surgery $(A)$ and mean cortisol levels after dexamethasone suppression test (DST) (B) are compared between subjects with PRKACA-mutant tumors (PRKACA) and subjects with mutations in other genes or no identified mutations (others). Shown are mean \pm s.D. PRKACA mutations are associated with younger age at surgery and higher cortisol levels after DST. (C) Number of patients with subclinical vs overt Cushing's syndrome in both groups. PRKACA mutations are associated with overt Cushing's syndrome. (D) Side of adenoma (left vs right) in tumors with CTNNB1 mutations vs tumors with other mutations or no identified mutations (others). CTNNB1 mutations are associated with right adenomas. ${ }^{*} P<0.05$ (Mann-Whitney $U$ tests (A), (B) or Fisher's exact test (C), (D)). See text for details.

described in pure aldosterone-secreting adenomas or hormonally inactive adrenal tumors $(9,22)$. Interestingly, both in this study and in a prior study (4), simultaneous mutations in GNAS and CTNNB1 were found in one tumor each, suggesting that cortisol production in these cases may be mainly caused by mutant GNAS. Of note, both these patients were male, and the mutation spectrum appears to be different in male vs female subjects (see below). PRKACA and GNAS mutations have so far been mutually exclusive in all studies (Supplementary Table 1), suggesting that mutation of either of the two genes is sufficient to cause autonomous cortisol secretion, in line with known physiology. Whether additional somatic mutations, germline alterations, or epigenetic factors determine the hormonal status of the remaining tumors with CTNNB1 mutations remains to be determined.

In line with a recent study from Japan (7), we do not observe PRKACA mutations in tumors co-secreting aldosterone and cortisol, suggesting that elevated PKA activity specifically upregulates cortisol secretion but not aldosterone secretion. However, tumors in the 'pure' cortisolsecreting adenoma group were not systematically screened for aldosterone co-secretion, therefore aldosterone secretion cannot be formally excluded in all.

We found KCNJ5 mutations in tumors predominantly secreting aldosterone, with concomitant cortisol secretion, again in line with prior work $(7,16)$. KCNJ5 mutations cause abnormal sodium permeability of the mutant potassium channel, leading to depolarization, activation of voltage-gated calcium channel, and calcium influx (19). It is conceivable that one or more of the signals downstream of calcium influx that have been implicated in aldosterone secretion are involved in cortisol production as well. Potential candidates include ATF2, CREB, and CREM that are activated by calmodulin kinases, but also act downstream of PKA (23). Consistent with this notion, an in vitro study using the HAC-15 human adrenocortical cancer cell line (24) showed slight upregulation of cortisol production after viral transduction with $K C N J 5^{T 158 A \text {, }}$ a mutation implicated in primary aldosteronism.

Both cortisol- and aldosterone-producing adenomas are more frequent in the left adrenal gland and are more prevalent in females than in males (18), yet the underlying pathophysiology remains unclear. In the case of aldosterone-producing adenomas, KCNJ5 mutations appear to fully account for the female preponderance (25), and this effect is not seen in patients with CACNA1D or ATPase mutations $(20,22)$. In our cohort, there were only three male individuals among the subjects with pure cortisolproducing adenomas, all with CTNNB1 mutations (in one case combined with a GNAS mutation), so it seems possible that a similar gender effect as for KCNJ5 mutations applies to PRKACA and tumors without known mutations. In our cohort, CTNNB1 mutations were more frequent on the right side, while overall, tumors were more frequent on the left side. Previous genetic studies have not reported tumor side; therefore, it would be interesting to see whether this effect is present in other cohorts as well.

Another interesting observation is the finding of a PRKACA mutation in unilateral adrenal hyperplasia with 
cortisol production, a rare phenotype that has not been studied in previous publications on PRKACA mutations. While a few cases of unilateral hyperplasia have been reported (reviewed by Takamura et al. (26)), typically, nodular hyperplasia was described. In contrast, macroscopic nodules were not detected in our case (Fig. 2). As demonstrated for ARMC5 mutations in ACTH-independent macronodular hyperplasia, the nodular structure in bilateral disease is typically caused by independent somatic mutations in individuals with underlying germline mutations. We suspect that a similar mechanism accounts for the individuals in our cohort who presented with recurrent and/or bilateral cortisol-producing adenomas, another rare entity (27). Pathology in these cases was not typical of ACTH-independent macronodular hyperplasia or primary pigmented nodular adrenal disease, and no germline mutation characteristics of these diseases were found. Future genome- or exome-level sequencing of additional cases may reveal the underlying pathophysiology.

In conclusion, we confirm the high frequency of PRKACA and CTNNB1 mutations in cortisol-producing adenomas and demonstrate that PRKACA mutations are associated with a more severe phenotype. For the first time, we identify the characteristic PRKACA ${ }^{L 206 R}$ mutation in a sample with diffuse unilateral hyperplasia. We suggest the presence of an unrecognized phenotype of adrenal Cushing's syndrome that features bilateral adenomas, possibly due to yet-unrecognized germline mutations. These samples warrant further studies.

\section{Supplementary data}

This is linked to the online version of the paper at http://dx.doi.org/10.1530/ EJE-14-1113.

\section{Declaration of interest}

The authors declare that there is no conflict of interest that could be perceived as prejudicing the impartiality of the research reported.

\section{Funding}

This work was supported by a grant of the Ministry of Innovation, Science, Research and Technology of the state of North Rhine-Westphalia, Germany (to U I Scholl). G Goh is supported by the Agency for Science, Technology and Research of Singapore. Part of the work received support by the Deutsche Forschungsgemeinschaft (grant number WI 3660/1-1 (KFO252) to H S Willenberg).

\section{Author contribution statement}

U I Scholl and G Goh designed the study; M Haase, H S Willenberg, $\mathrm{M}$ Schott, and $\mathrm{U}$ I Scholl evaluated subjects and ascertained clinical information; A-C Reis evaluated histological specimens and provided tissue cores; A Thiel performed Sanger sequencing and statistical analysis; and A Thiel and $U$ I Scholl wrote the manuscript, with all authors contributing to a critical revision.

\section{Acknowledgements}

The authors thank their patients for their invaluable contribution to this project, Irina Tikhonova, the Yale Center for Genome Analysis, the Düsseldorf Center for Advanced Imaging (CAi, Steffen Köhler), and the Düsseldorf and Essen Pathology Laboratories for support. They also acknowledge members of the Düsseldorf Departments of Medicine and Surgery involved in patient care, and L Christian Rump, Helmut E Gabbert, and Kurt W Schmid for providing laboratory space and support.

\section{References}

1 Kirschner LS. Medicine. A unified cause for adrenal Cushing's syndrome. Science 2014344 804-805. (doi:10.1126/science.1254901)

2 Orth DN. Cushing's syndrome. New England Journal of Medicine 1995 332 791-803. (doi:10.1056/NEJM199503233321207)

3 Beuschlein F, Fassnacht M, Assie G, Calebiro D, Stratakis CA, Osswald A, Ronchi CL, Wieland T, Sbiera S, Faucz FR et al. Constitutive activation of PKA catalytic subunit in adrenal Cushing's syndrome. New England Journal of Medicine 2014370 1019-1028. (doi:10.1056/ NEJMoa1310359)

4 Cao Y, He M, Gao Z, Peng Y, Li Y, Li L, Zhou W, Li X, Zhong X, Lei Y et al. Activating hotspot L205R mutation in PRKACA and adrenal Cushing's syndrome. Science 2014344 913-917. (doi:10.1126/science.1249480)

5 Sato Y, Maekawa S, Ishii R, Sanada M, Morikawa T, Shiraishi Y, Yoshida K, Nagata Y, Sato-Otsubo A, Yoshizato T et al. Recurrent somatic mutations underlie corticotropin-independent Cushing's syndrome. Science 2014344 917-920. (doi:10.1126/science.1252328)

6 Goh G, Scholl UI, Healy JM, Choi M, Prasad ML, Nelson-Williams C, Kunstman JW, Korah R, Suttorp AC, Dietrich D et al. Recurrent activating mutation in PRKACA in cortisol-producing adrenal tumors. Nature Genetics 201446 613-617. (doi:10.1038/ng.2956)

7 Nakajima Y, Okamura T, Gohko T, Satoh T, Hashimoto K, Shibusawa N, Ozawa A, Ishii S, Tomaru T, Horiguchi K et al. Somatic mutations of the catalytic subunit of cyclic AMP-dependent protein kinase (PRKACA) gene in Japanese patients with several adrenal adenomas secreting cortisol [Rapid Communication]. Endocrine Journal 201461 825-832. (doi:10.1507/endocrj.EJ14-0282)

8 Di Dalmazi G, Kisker C, Calebiro D, Mannelli M, Canu L, Arnaldi G, Quinkler M, Rayes N, Tabarin A, Laure Jullie M et al. Novel somatic mutations in the catalytic subunit of the protein kinase A as a cause of adrenal Cushing's Syndrome: a European multicentric study. Journal of Clinical Endocrinology and Metabolism 201499 E2093-E2100. (doi:10.1210/jc.2014-2152)

9 Berthon A, Martinez A, Bertherat J \& Val P. Wnt/ $\beta$-catenin signalling in adrenal physiology and tumour development. Molecular and Cellular Endocrinology 2012351 87-95. (doi:10.1016/j.mce.2011.09.009)

10 Tadjine M, Lampron A, Ouadi L \& Bourdeau I. Frequent mutations of $\beta$-catenin gene in sporadic secreting adrenocortical adenomas. Clinical Endocrinology 2008 68 264-270. (doi:10.1111/j.1365-2265.2007.03033.x)

11 Berthon A, Sahut-Barnola I, Lambert-Langlais S, de Joussineau C, Damon-Soubeyrand C, Louiset E, Taketo MM, Tissier F, Bertherat J, Lefrancois-Martinez AM et al. Constitutive $\beta$-catenin activation induces adrenal hyperplasia and promotes adrenal cancer development. Human Molecular Genetics 201019 1561-1576. (doi:10.1093/hmg/ddq029)

12 Fragoso MC, Domenice S, Latronico AC, Martin RM, Pereira MA, Zerbini MC, Lucon AM \& Mendonca BB. Cushing's syndrome secondary to adrenocorticotropin-independent macronodular 
adrenocortical hyperplasia due to activating mutations of GNAS1 gene. Journal of Clinical Endocrinology and Metabolism 200388 2147-2151. (doi:10.1210/jc.2002-021362)

13 Magiakou MA, Smyrnaki P \& Chrousos GP. Hypertension in Cushing's syndrome. Best Practice \& Research. Clinical Endocrinology \& Metabolism 200620 467-482. (doi:10.1016/j.beem.2006.07.006)

14 Cicala MV \& Mantero F. Hypertension in Cushing's syndrome: from pathogenesis to treatment. Neuroendocrinology 201092 (Suppl 1) 44-49. (doi:10.1159/000314315)

15 Willenberg HS, Spath M, Maser-Gluth C, Engers R, Anlauf M, Dekomien G, Schott M, Schinner S, Cupisti K \& Scherbaum WA. Sporadic solitary aldosterone- and cortisol-co-secreting adenomas: endocrine, histological and genetic findings in a subtype of primary aldosteronism. Hypertension Research 201033 467-472. (doi:10.1038/ hr.2010.18)

16 Yamada M, Nakajima Y, Taguchi R, Okamura T, Ishii S, Tomaru T, Ozawa A, Shibusawa N, Yoshino S, Toki A et al. KCNJ5 mutations in aldosterone- and cortisol-co-secreting adrenal adenomas [Rapid Communication]. Endocrine Journal 201259 735-741. (doi:10.1507/ endocrj.EJ12-0247)

17 Spath M, Korovkin S, Antke C, Anlauf M \& Willenberg HS. Aldosteroneand cortisol-co-secreting adrenal tumors: the lost subtype of primary aldosteronism. European Journal of Endocrinology 2011164 447-455. (doi:10.1530/EJE-10-1070)

18 Kumar V, Abbas AK, Fausto N \& Aster C. In Robbins and Cotran Pathologic Basis of Disease. Philadelphia: Saunders, 2009.

19 Choi M, Scholl UI, Yue P, Bjorklund P, Zhao B, Nelson-Williams C, Ji W, Cho Y, Patel A, Men CJ et al. $\mathrm{K}^{+}$channel mutations in adrenal aldosterone-producing adenomas and hereditary hypertension. Science 2011331 768-772. (doi:10.1126/science.1198785)

20 Beuschlein F, Boulkroun S, Osswald A, Wieland T, Nielsen HN, Lichtenauer UD, Penton D, Schack VR, Amar L, Fischer E et al. Somatic mutations in ATP1A1 and ATP2B3 lead to aldosterone-producing adenomas and secondary hypertension. Nature Genetics $2013 \mathbf{4 5}$ 440-444. (doi:10.1038/ng.2550)

21 Azizan EA, Poulsen H, Tuluc P, Zhou J, Clausen MV, Lieb A, Maniero C, Garg S, Bochukova EG, Zhao W et al. Somatic mutations in ATP1A1 and CACNA1D underlie a common subtype of adrenal hypertension. Nature Genetics 201345 1055-1060. (doi:10.1038/ng.2716)

22 Scholl UI, Goh G, Stolting G, de Oliveira RC, Choi M, Overton JD, Fonseca AL, Korah R, Starker LF, Kunstman JW et al. Somatic and germline CACNA1D calcium channel mutations in aldosteroneproducing adenomas and primary aldosteronism. Nature Genetics 2013 45 1050-1054. (doi:10.1038/ng.2695)

23 Dringenberg T, Schwitalla M, Haase M, Scherbaum WA \& Willenberg HS. Control of CYP11B2/CYP11B1 expression ratio and consequences for the zonation of the adrenal cortex. Hormone and Metabolic Research 201345 81-85. (doi:10.1055/s-0032-1331210)

24 Oki K, Plonczynski MW, Luis Lam M, Gomez-Sanchez EP \& GomezSanchez CE. Potassium channel mutant KCNJ5 T158A expression in HAC-15 cells increases aldosterone synthesis. Endocrinology 2012153 1774-1782. (doi:10.1210/en.2011-1733)

25 Akerstrom T, Crona J, Delgado Verdugo A, Starker LF, Cupisti K, Willenberg HS, Knoefel WT, Saeger W, Feller A, Ip J et al. Comprehensive re-sequencing of adrenal aldosterone producing lesions reveal three somatic mutations near the KCNJ5 potassium channel selectivity filter. PLoS ONE 20127 e41926. (doi:10.1371/journal.pone.0041926)

26 Takamura T, Nagai Y, Taniguchi M, Yamashita H, Nakamura S, Ikeda T, Kobayashi K, Suzuki T \& Sasano H. Adrenocorticotropin-independent unilateral adrenocortical hyperplasia with Cushing's syndrome: immunohistochemical studies of steroidogenic enzymes, ultrastructural examination and a review of the literature. Pathology International 200151 118-122. (doi:10.1046/j.1440-1827.2001.01169.x)

27 Dinneen SF, Carney JA, Carpenter PC, Grant CS \& Young WF Jr. Acthindependent Cushing's syndrome: bilateral cortisol-producing adrenal adenomas. Endocrine Practice 1995 17-81. (doi:10.4158/EP.1.2.77)

Received 14 December 2014

Revised version received 13 February 2015

Accepted 6 March 2015 